\title{
A unified framework for political parties to support e-democracy practices: the case of a Greek party
}

\section{Bouras* and E. Giannaka}

Research Academic Computer Technology Institute and Computer Engineering and Informatics Dept., University of Patras, Nikolaou Kazatzaki Str., Patras University Campus, 265 00, Rion, Greece Fax: +30-2610-960358 Fax: +30-2610-960380

E-mail: bouras@cti.gr_E-mail: giannaka@cti.gr

${ }^{*}$ Corresponding author

\section{Th. Karounos}

School of Electrical and Computer Engineering, Network Management and Optimal Design Laboratory (NETMODE),

National Technical University of Athens,

9 Iroon Polytechniou Str., 15780 Athens, Greece

Fax:+30-210-7721452 E-mail: karounos@pasok.gr

\section{A. Priftis}

Department of Information and Communication Systems Engineering, University of Aegean, AegeaNet, Emporiki, GR 83200 Karlovassi, Samos, Greece

E-mail: priftis@pasok.gr

\section{Poulopoulos}

Research Academic Computer Technology Institute and Computer Engineering and Informatics Dept., University of Patras, Nikolaou Kazatzaki Str., Patras University Campus, 265 00, Rion, Greece Fax:+30-2610-960358 E-mail: poulop@ceid.upatras.gr

\section{Th. Tsiatsos}

Department of Informatics, Aristotle University of Thessaloniki \& Research Academic Computer Technology Institute - Research Unit 6, P.O. Box 114, GR-54124, Thessaloniki, Grece

Fax:+302310998419 E-mail: tsiatsos@csd.auth.gr 


\begin{abstract}
E-government and e-democracy constitute a central theme in information society policy at all levels: local, regional, national, European and even globally. To this direction, a variety of efforts have been made, and many systems have been developed. This paper proposes a methodology for designing and implementing web-based services that can support e-democracy practices. Furthermore, the paper presents and describes the effort made by a Greek political party to this direction, for the design and development of a unified framework for providing, supporting and managing e-democracy services. The paper describes the actions taken and the work done according to the proposed methodology.
\end{abstract}

Keywords: e-government; e-democracy; open source software.

Reference to this paper should be made as follows: Bouras, C., Giannaka, E., Karounos, Th., Priftis, A., Poulopoulos, V. and Tsiatsos, Th. (2008) 'A unified framework for political parties to support e-democracy practices: the case of a Greek party', Int. J. Electronic Democracy, Vol. 1, No. 1, pp.98-117.

Biographical notes: Christos Bouras obtained his Diploma and $\mathrm{PhD}$ from the Computer Science and Engineering Department of Patras University, Greece. $\mathrm{He}$ is currently an Associate Professor in the above department. Also, he is a Scientific Adviser of Research Unit six in Research Academic Computer Technology Institute (CTI), Patras, Greece. His research interests include analysis of performance of networking and computer systems, computer networks and protocols, telematics and new services, QoS and pricing for networks and services, e-learning, networked virtual environments and www issues. He has extended professional experience in design and analysis of networks, protocols, telematics and new services.

Eri Giannaka obtained her Diploma from the Informatics Department of the Aristotelian University of Thessaloniki (Greece) and her Master's Degree from the Computer Engineering and Informatics Department of Patras University. She is currently a PhD Candidate of the Department of Computer Engineer and Informatics of Patras University. Furthermore, she is working as an R\&D Computer Engineer at the Research Unit six of the Computer Technology Institute in Patra, Greece. Her interests include virtual reality applications, performance evaluation of networked virtual systems, algorithms and techniques for performance optimisation in virtual environments, design and implementation of virtual worlds, distributed systems, internet applications, database implementation and administration and programming.

Theodoros Karounos studied in the Technical School of Sivitanidios and received his $\mathrm{BSc}$ in Computer Science and the MSc in Information and Communication Systems. He was employed by IBM, New York, USA, as a Systems Analyst. He worked as a coordinator for R\&D at the Informatics Development Agency of the Greek Ministry of Presidency and as an Adviser for Informatics to the Greek Minister of the Interior. Currently, he is Teaching Assistant at the Network and Optimal Design Laboratory - NETMODE $<$ http://www.netmode.ntua.gr/> of the Department of Electrical and Computer Engineering of NTUA and Coordinator of ICT at PASOK (Greek Socialist Party).

Athanasios Priftis is a $\mathrm{PhD}$ candidate on Information Society, e-governance and e-democracy issues at the Aegean University (Greece). He holds an MSc in Information Science from University of Loughborough (UK). $\mathrm{He}$, currently, works as an IT Analyst for PASOK's (Greek Socialist Party) Internet Technologies Department aiming to bring citizens closer to its content 
and services through the use of Free and Open Source Software (FOSS) methodologies both on the organisational and production level as well as the one of the internet service provisions.

Vassilis Poulopoulos entered the Computer Science and Engineering Department of Patras University, Greece in 2000. He obtained his Diploma on July 2005 and his MSc on September 2007. He is a member of Research Unit 6 since December 2001. His basic fields of interest are web services, web components, web technologies, web integration, web content manipulation, text pre-processing, categorisation and summarisation, web personalisation and programming. He has expertise on issues regarding technologies and services of Web 2.0. He has extended knowledge of the following programming languages: ASP, PHP and JSP language programming, HTML programming, $\mathrm{C}, \mathrm{C}++$ and Java programming and database manipulation.

Thrasyvoulos Tsiatsos obtained his Diploma, his Master's Degree and his $\mathrm{PhD}$ from the Computer Engineering and Informatics Department of Patras University, Greece. He is currently Lecturer in the Department of Informatics of Aristotle University of Thessaloniki as well as research member at the Research Unit six of Research Academic Computer Technology Institute. His research interests include computer networks, telematics, networked virtual environments, multimedia and hypermedia. More particular, he is engaged in Distant Education with the use of computer networks, real-time protocols and networked virtual environments. He has published more than 40 papers in journals and in well-known refereed conferences and he is co-author in two books.

\section{Introduction}

Nowadays, the internet is of high importance, as it constitutes one of the basic means for communication, training and collaboration among individuals through the exchange of ideas and knowledge. The wide adoption of this powerful medium and the familiarisation by enterprises, citizens and individuals with it was sought for many public authorities as an important step forward in availability and accessibility, as it could provide and support access to information along with a certain degree of interaction among the citizens and the authorities involved. In particular, both the internet and the current state of Information and Communication Technologies (ICT) allow public authorities to adopt a citizen-oriented approach, providing public services organised around their centres of interests (Garcia-Arribas and Lopez-Crespo, 2003). Furthermore, apart from the existence of the internet as the basic medium for communication, diffusion of knowledge and interaction among the authorities and the individuals, e-government and e-democracy also presuppose that the services provided and the information management is supported by efficient and reliable information systems as well as by a back office, which enables public administrations to interact electronically with individuals. As stated in Pizarro and De La Torre, these presuppositions are based on another important hypothesis:

"online administrative procedures are subject to sets of explicit rules, based on standards, which are systematically applied with no scope for arbitrariness or discretion in their application." (Pizarro and De La Torre, 2003) 
As far as it concerns e-government, it should be mentioned that it is a tool not a goal itself and it should, therefore, assist the delivery of better government in the following directions (Liikanen, 2003):

- E-government should make it possible for citizens to follow their governments, central, regional and local actions, to be able to participate in decision-making. This is about increasing democratic participation and involvement.

- E-government should help to provide citizens personalised public services that meet their specific needs.

- E-government should increase efficiency and productivity. The public sector, as an information-intensive sector, can be made more efficient by digitising information and processes.

Regarding e-democracy (Poullet, 1998), this field supports a variety of ways for political participation, from access to public information via formal and informal participation in planning and decision-making processes, to online voting in elections (Kubicek et al., 2003). For the exploitation of the benefits that could be extracted by this approach, the provision of services and facilities, from a technical point of view, cannot succeed. Rather,

"technology has to be embedded into organisational, social, legal and cultural changes, it has to be integrated into political and administrative processes on the side of the institutions which offer participation and has to be matched with the interests of the people addressed." (Kubicek et al., 2003)

As stated in Musgrave (2004), the use of computers and IT by central, regional, and local government departments has shifted from being an administration tool to become a significant part of the overall service delivery mechanism, and a key component of the reform agenda. In particular, e-government and e-democracy constitute a central theme in information society policy at all levels: local, regional, national, European and even globally. To this direction, a variety of efforts have been made and many systems have been developed. The majority of these efforts are found to be political parties across Europe, which adopt these approaches for communicating with the citizens and for promoting their participation on political issues. At this point, it should be mentioned that political parties, apart from their political activities, operate also as informational organisations, which collect, organise, promote and administer information. Therefore, there is a strong need for these parties to maintain the appropriate tools and services for all of its members (i.e., the President, the Members, the Deputies, etc.) both in a national and European level (the officers, the employers to the members and the friends), so that they can collaborate, participate in the processes and activities of the party and become more effective. These tools and processes could allow all representatives to get informed, participate, and communicate both with the other representatives as well as with citizens, collaborate more effectively and adopt a variety of new services.

In this paper, such an effort made from a Greek party is presented. In particular, this paper is engaged with the description of the steps that were followed for the development of a unified framework for supporting e-democracy (e-PASOK) by the party of PASOK. The objectives of this effort were twofold: on the one hand, the development of a system that, based on the internet, could form a communication medium between the party 
and the citizens was addressed and on the other hand the adoption of a system, as a supplementary medium, for the support of the internal processes and actions within the party was sought.

The remainder of this paper is structured as follows. In Section 2, related efforts from other political parties in the direction of e-government and e-democracy are presented, while in Section 3 a proposed methodology for designing e-services for political parties and which is adopted for the realisation of e-PASOK is described. Section 4 is dedicated to the case of PASOK, in terms of the motivation behind the effort made, its vision of the developed system, the benefits expected from its adoption for the realisation of e-democracy and the assessment of the current state of the methods and actions performed in the party. Following this, the functional specifications, in terms of the unified administrative framework and the services provided, are presented. Section 6 continues with the technical specifications of the developed system, regarding the technological approach adopted and the system architecture decided. Finally, some concluding remarks and planned next steps are briefly described.

\section{E-democracy services and political parties}

Many countries, especially the more developed in the field of networks and generally network technologies, have made remarkable attempts to organise their domestic offices and their parties. The use of intranet may lead to increase in productivity and efficiency of the employees, better classification of an organisation, simplification of actions and procedures, and to better organisation of the information (Poullet, 1998).

Some of the organisations that have adopted e-services in an optimised way are the following:

- Defense Finance and Accounting Service in USA (http://www.dfas.mil)

- Department of Transport in UK (http://www.dft.gov.uk)

- Department of Victorian Communities in Australia: (http://www.dvc.vic.gov.au)

- Government Offices in Sweden (http://www.sweden.gov.se/).

The basic points that lead them to success are:

- Definition of the number of people who will participate. Absolute definition of the participant, which lead to absolute evaluation of the departments and to almost perfect tracing of the procedures and flows.

- Encouraging the users to exchange information through the system. Good management of the users, especially of those that use the system more often. It is very important to support the users and even train them for a long period of time to make them familiar with the new system.

- Central Information Management. The information in the aforementioned systems was centralised, collected to a central database. Consequently, the system was stable, flexible and always up-to-date. 
- Use of Content Management System (CMS). All the transferred information, whose size may be enormous, must be supported to be a CMS. This system will be responsible for the good organisation of the information and it will attach roles to each user regarding access to information and functionality restriction.

- $\quad$ Avoid activity overlapping. To achieve the avoidance of overlapping, which increases productivity, the system should have the functionality to inform each user, of each section of the organisation, for its responsibilities, and for the responsibilities of the other users on a specific flow.

- $\quad$ Provide help to the lower sections. The hierarchy in an organisation is the element that helps the employees to realise their responsibilities and their authorities. Apart from that, the hierarchical model leads to a system where employees in lower section can ask for help from the employees of higher sections. This action is leading to a good practice through which the higher sections make extended use of any new installed system to motivate all the employees of the organisation to use the system.

- Unified Technology. The section of the technology used for the aforementioned systems does seem to be an important issue. Furthermore, these systems use unified technology based on open standards.

- Test before use. As in every informational system, a full check and inspection of the system must be done before the system is on the air. In many cases, the testing procedure lasted for more than six (6) months to produce a system with high security standards.

All the above lead to the extraction of the following conclusions on the metrics that were applied on the aforementioned systems:

- Increase in productivity, decrease in functional cost. In some cases, the increase in productivity was $300 \%$, an extremely huge number for a low-cost procedure, which is the installation of a system. In some cases, the decrease in the functional cost reached unrealistic numbers. An example can be the Postal Savings Bank of Greece where the installation of an intranet for the internal communication leads to a decrease in the function cost to an amount of $10,000 \%$.

- Better organisation, reduction of information loss: In general, a centralised information system can give impulse to an organisation. In the aforementioned systems, after a short period of time, reports were written announcing that many positive points were observed. In addition, something very important was pointed out that the information loss had been reduced.

The aforementioned cases are just few of the successful in the world concerning intranet. The analysis of these systems can be used as a guide for any attempt for construction of an intranet-based system. Besides, the aforementioned systems are until now 'up and running' with great success and with increasing number of users and must become models for further spreading of automation through organisations. 
The basic tensions can be summarised to three points:

- the use of technology through political procedures can have positive results

- the internet, intranet and World Wide Web are a normal way of producing and enforcing democracy

- one of the forms of making politics and producing valid critics through the internet and intranet is creating and expressing papers and publishing them into key web portals.

\section{Proposed methodology}

This section is dedicated on describing shortly a proposed general methodology for designing and adopting e-services for political parties.

It has to be realised that the technologies will not in themselves cause a technologically enabled change to happen (Benjamin and Levinson, 1993). New technology is not enough to increase productivity. Organisational and process changes must also take place.

Thus, changes from the current state are inspired by a vision. The vision is used to motivate and based on this motivation a level of transformation is reached. The transformation may be a transitory step on the way to accomplishing the overall vision.

The requirements of the management model for transition of government using information and telecommunication technologies are both complex and far reaching. To effect the levels of change, it is necessary to accommodate all of these aspects, and it requires the use of a very comprehensive methodology (Figure 1).

Figure 1 Proposed methodology (see online version for colours)

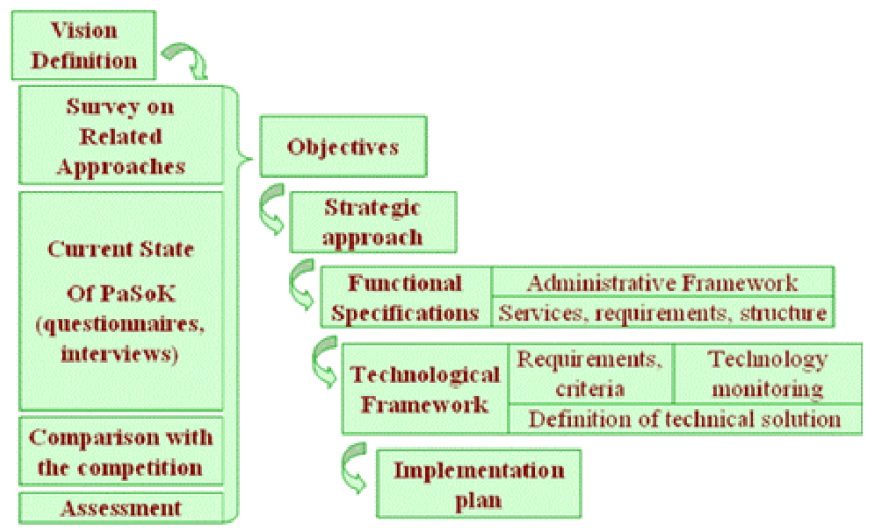

After defining the vision, the next step is the research and survey of the current status within the party as well as on its needs, the analysis of better practices and the bibliographic research. After tracing the current status, an assessment is required to define the strengths, weaknesses, opportunities, threats (SWOT analysis) to define realistic objectives. 
The next step in the proposed methodology is to define a general strategic approach for applying the change in the party concerning the transition from traditional services to the provision of e-services. This strategic approach should include business process reengineering, organisational structure change, organisational culture and organisational learning.

Based on the results extracted from the survey of the current status of the parties, the objectives and the strategic approach, the basic components of the technological system, in terms of functional specifications should be defined.

After that, we propose to define the technical requirements and the criteria for the selection of the technologies as well as to elaborate a technical state of the art to conclude in a definition of a viable technical solution.

The final step to be defined is an implementation plan, which should be appropriate according to the strategic approach and the priorities defined by the party.

The above-described methodology has been applied in the case of one of the major Greek political parties, PASOK. This process is described in the remainder of this paper.

\section{The case of PASOK}

PASOK (http://www.pasok.gr, http://en.wikipedia.org/wiki/PASOK) is a political party - organisation that is changing itself to facilitate an effective dialogue between politicians and citizens. The first step was taken with the launching of a PASOK National Dialogue, aiming to enhance the party's political platform that lasted three months and involved thousands of people throughout the country.

PASOK, through its political leadership, is realising the complexity and the different challenges regarding the development of e-democracy: educational, technological and political. Based on the above as well as on the fact that both electronic communication and the information administration could significantly contribute to the improvement of the realisation of the processes that individuals, within the party, perform in regard to their needs for access to information, communication and collaboration, PASOK proceeded with the development of an informational system, which aims at the facilitation of the information circulation and disposal within the boundaries of PASOK as well as the encouragement of the communication, participation and collaboration among its friends, its members and its officers.

Furthermore, PASOK recognising the potentialities of the internet aims to exploit it systematically as an effective medium of constant informing, permanent and bidirectional communication, open dialogue and organised public deliberation with its members and friends, with the forces of knowledge and enlightenment, the social and union organisations as well as, in a wider range, with the citizens of Greece. The internet will constitute for the PASOK not only a medium of organisation, communication, participation and education, but also the unique medium that will provide all of the above. The internet can form a powerful tool for PASOK, as an open and participative party, for the exchange of ideas and knowledge, their further deliberation through an electronic floor for dialogue and the access to them in many different forms. The internet should form a tool for the construction, organisation, communication, participation, deliberation and actuation, within the frame of the constitution of a modern open party that believes in the transparency of the positions and the processes as well as in the wide participation of the citizens, thus completing, without though substituting, other, more 
traditional forms of communication and political practice. In addition, this tool, through its paradigmatic operation, will affect both the overall operation of the national institutions and the way that the citizens are activated.

The main motivation behind this effort could be summarised as follows:

- quick and reliable access to elaborated information, which mainly concerns documents, literature, Greek and International Press, interviews, etc.

- access to various forms of information, such as audiovisual clips as well as documents

- support of a dialogue rostrum for promoting the collaboration and participation in decision-making and movement assessment.

The following paragraphs are describing the work done by PASOK for providing a Unified Framework for the Collection, Management and Disposition of Content as well as for e-democracy, called e-PASOK, according to our proposed methodology.

\subsection{Vision}

In short, the vision of PASOK, regarding its participation in the electronic government of Greece, is the following:

"In the next three years the party of PASOK will constitute a political party paradigm and model in the exploitation of ICT for the improvement of the performance, effectiveness, thoroughness and diffusion of the services that it will provide to its officers, members and the citizens, by providing and supporting complete and advanced services, with informational and interactive characteristics, and thus achieving the optimisation of its resources management, the increment of the productivity and the transparency of its processes and activities."

As it becomes clear from the above, e-PASOK is an important investment for the future for the party of PASOK. In particular, it will be an informational system, based on the World Wide Web and will constitute for the party of PASOK (Figure 2):

Figure 2 The four axes (see online version for colours)

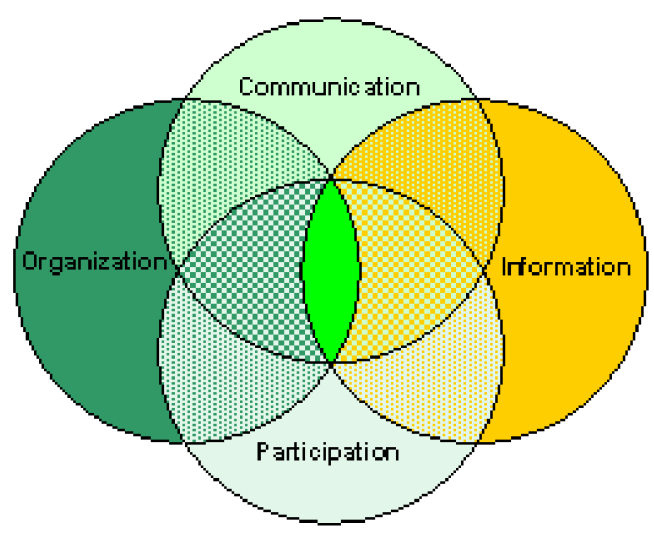


- A communication medium for the President, the Officers, the Members, the Friends, the Local Authorities, the Prefecture Committees, the Members of the Parliament and the European parliament. e-PASOK will contribute with new ways of communication, such sms messages to, forums, real-time communication, the internet (chat, tele-conference), etc.

- A participation medium for everyone, independently from his or her geographical location: e-PASOK will provide new ways of participation in the activities and processes of an open party, as the possibility of microsites creation of the Local Authorities, the Prefecture Committees of Education, the Officers, the Members of Parliament and the Euro-Parliament.

- An information space, and a training medium for everyone, without prohibitions. e-PASOK will constitute the personalised access point for the Officers, the Friends, the Members and the voters of the party.

- A tool for the organisation of the party and for supporting its operations. e-PASOK will constitute an important tool for the party of PASOK, and it will impulse it by improving its structure and minimising the loss of information and data. Processes, which are currently time-consuming, will be computerised so as the effectiveness of the party will be improved. In addition, e-PASOK will support the employees in their every-day tasks by contributing in the cooperation of the different sectors in the handling of reports safely, quickly and effectively.

PASOK is a major political party as the number involved in its local and central departments, MPs, supporters and so on reach 1000,000. Therefore, the benefits that will arise for the party, as an organisation, from the exploitation of e-PASOK are foreseen to be multi-fold. In particular, this new system will contribute on the increment of the productivity, decrement of the operational costs, better organisation, minimisation of lost information, direct and valid training of the Officers, Employees, Members and Friends.

\subsection{Assessment of current state in PASOK}

The next step according to the previous described methodology is based on the research and survey of the current status within the party as well as on its needs, the analysis of better practices and the bibliographic research.

The survey of the current state constitutes one of the basic steps for designing a system, as it summarises the abilities of the party to develop and mainly support such a unified informational system. The method adopted is called SWOT ("Strengths, Weaknesses, Opportunities, Threats"), which defines the weaknesses, the strengths and the opportunities from the internal part of PASOK and the opportunities and threats are localised from the external part of the party.

For the realisation of the earlier method, questionnaires were formed and sent out to the employees of the party, in a Managerial and Sector level. This action formed the primer step for the collection of both data and proposals for the creation of an effective and usable system that, based on the internet, will provide information services to all in real time. The step that followed the questionnaires was the conduction of interviews with the basic sectors of the party, in terms of the individuals related with the production and diffusion of information. These interviews lasted for about three weeks. 
The results of the questionnaires and interviews performed have been elaborated according to SWOT analysis, and they are presented in the following sections.

Regarding the strengths, the results extracted are the following:

- Definite political intention from the President's side for the development, application and use of new technologies.

- Clear view of the responsible persons of the adoption of e-services for the needs and priorities regarding the development of electronic services.

- There is a strong tension in PASOK for the exploitation of Open Source Software and standards, which will assist the cost-effective and scalable development of an informational system.

- There is a 'necessary critical mass' and the content for the operation of a networked informational system.

- A technical and networking infrastructure is available as well as a relative know-how from the majority of the employees. The users, in the majority, seem to be familiar with new technologies and the use of computers.

- An Information Centre and an Internet Department is available.

- Extensive use of computers for the accomplishment of every-day tasks.

- The majority of the employees have a connection to the internet.

- A large number of users have access to an intranet.

- There is large number of the employees within the party of PASOK that have clear view on the applications they would like to use.

- There is a large number of scattered sites.

- $\quad$ There are some isolated efforts from some departments of PASOK for developing e-services.

Regarding the weaknesses of the current status within the party of PASOK, the results extracted could be summarised as follows:

- The current electronic equipment (computers, printers, scanners, etc.) is inadequate.

- There is need for the exploitation of common and unified applications in areas related with databases, files and Citizen Relationship Management (CRM) applications. The employees overlook for horizontal association of the sectors/departments in an intranet but they also find necessary the creation or purchasing of a programme that will allow the easy and quick diffusion and sharing of information.

- There is an urgent need for a unified users' management application.

- There is a segmental flow of information, limited cooperation processes and activities among the sectors and important problems in the diffusion of common information among interrelated sectors. 
- A generalised computer network should be designed and developed, which will be attended by the corresponding processes, flows and utilisation primitives.

- The training and participation of the executives and employees of concerning e-services PASOK is needed.

- There is a large number of scattered sites.

- There is a lack of an aggregated computer network.

- A large number of the informatics applications as well as the networked sites, currently in use, have not been developed under the same technological platform.

As far as it concerns the opportunities that are extracted both from the questionnaires and interviews conducted, the results could be summarised as follows:

- Introduction of Open Source Software.

- Increment of the exploitation of the internet.

- Familiarisation of the citizens with electronic services, as these are provided from the Supporting Citizens Services.

- Citizen training with parallel programmes from the Political Training Centre of PASOK.

- Training of a large percent of the civil servants to new technologies.

- Interconnection possibility with other (external) informational systems, such as the new informational system of the Parliament, through the appropriate interface.

Finally, as far as it concerns the threats that were extracted, these could be summarised as follows:

- citizens' denial to use of services owing to lack of the technological know-how and fear of diffusion of their personal information

- risk of weakness in the adaptation of the citizens in the new applications.

Summarising, Figure 3 presents a categorisation of PASOK's achievements in four (4) different phases:

- Online presence phase. The internet presence is limited to general information for the organisation through a website.

- Interaction phase. Enriched content is provided to the citizens, the ability to search and retrieve documents of their interest as well as the ability of a limited communication, which is mainly achieved via the e-mail.

- Transaction phase. Additional abilities are provided as registration, and enhanced capabilities of communication and collaboration through forums, free of the physical presence of the citizens.

- Transformation phase. Through a unique entry point (portal), the citizens, the employees and the executives of the organisation have the ability to communicate, collaborate and interact. 
Most of the parties in Greece maintain a website, and are currently in the 'Online Presence' phase. The number of the parties that stand in the 'Interaction' phase is small and the abilities for communication that they provide is limited. PASOK is currently at the 'Transformation' phase having already developed a variety of communication services with the citizens and in a way has completed the 'Transaction' phase. Based on the fact that the 'Transformation' phase requires important changes throughout the organisation, PASOK, apart from the adaptation of new technologies, aims also at the transformation of the internal processes.

Figure 3 SOK at the transformation doorstep (see online version for colours)

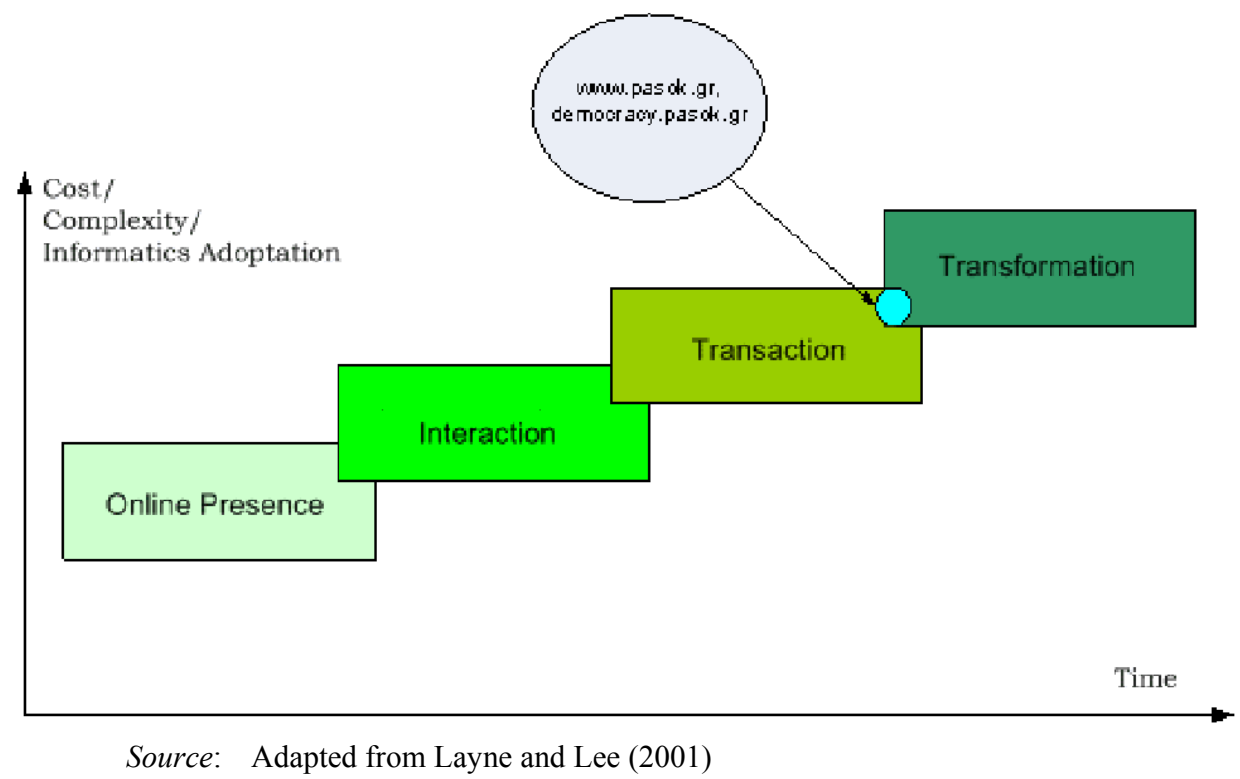

At this point, it should also be mentioned that in the current state, PASOK is engaged with projects, which are aiming at:

- Creation of a digital solidarity. Recruiting internet volunteers that are educating party cadre on the basics of internet. Publishing a "guide on internet's political use. The $\mathrm{ABC}$ of internet for political activists" analysing the possibilities and difficulties of internet's political use.

- Opening up the civic space. The e-democracy project (democracy.pasok.gr), which fosters grassroots dialogue engaging 6000 registered users and 20,000 unique visitors per month, during the PASOK National Dialogue, in an open participation platform that resulted to 550 written contributions and over 2000 forum posts on various issues.

Finally, there is also a major internet news site running, covering all PASOK news and activities, with 40,000 unique visitors per month (www.Pasok.gr). From the above, it becomes clear that there is a critical mass and the willing for the transition to e-democracy practices. 


\subsection{Objectives}

According to the aforementioned methodology, it is fundamental to explicitly define the objectives of the party of PASOK for the new informational system to be developed, based on the World Wide Web. These objectives are the following:

- Global presence. There are no more time and spatial limitation for the realisation of the processes and the activities of the movement.

- Accessibility through the friendly disposition of information. Both the already prepared and generated information will be easily available and accessible for the users through the World Wide Web.

- Quick access to information, through instant updating and transmission of information.

- Personalisation. Through the exploitation of personalised services, there will be the possibility for the adaptation of the way that the information will be accessible from each user.

- Training for everyone, through the wider diffusion of information, advising and updating.

- Collaboration-Participation. Encouragement of the collaboration among the members of the party through the instant and friendly access to processes and participation activities in the frame of distant dialogue, with both synchronous and asynchronous ways. The wider objective is the modulation of a new progressive political premise for Greece.

- Transparency. Through the access to information and participation with the transparent and effective operation of an open progressive political organisation, of the reconstructed PASOK.

\subsection{Strategic approach}

The strategic approach implies the need for a set of processes that need to be performed for the digitalisation of the content and the transformation of services to 'electronic' form. These processes involve the collection of the available information, whether electronic or not, the digitalisation of each generated information as well as its management through the informational system. The process that finalises the procedure is the publication and provision of the above-mentioned information. Therefore, certain structures and models as well as flows need to be clearly identified for the definition of the technological framework, upon which the system development will be realised. At this point, it should be mentioned that an important part of the strategic approach analysis need to be focused on the registration and assessment of the internal processes, the determination of the data flows available and the preparation of the human resources so as the adaptation of the system will be feasible.

From the above, it becomes clear that the objectives of the developed framework for the management should be the following: 
- Creation of a certain group of users, who will guide, educate, train and assist the users of the system so as the accession of the newborn environment will be achieved.

- Definition of the processes that need to be followed for the assessment of the users' actions so that consistency in the collection, management and diffusion of the generated information to be achieved.

- Description of a technological basis, on top of which the system development will be based for the achievement of the strategic objectives.

The application of the above is found critical for the design of the necessary infrastructure, on top of which the system development will be based.

\section{Functional specifications}

Based on the results extracted from the survey of the current status of PASOK, the basic components of e-PASOK have been defined, in terms of the Unified Administrative Framework of the Informational system, the services and requirements of the structure of the web-based system.

\subsection{Unified administrative framework}

Regarding the Unified Administrative Framework of the Informational System, it was important to define and describe the processes and necessary components from the initialisation of the developed system until the final stage of the information disposal. In particular, for the end user the system constitutes a set of services, each of which can support certain functionality. The user, in regard to his/her access rights, can realise certain actions and exploit either a subset or all of the provided functionalities, which form a service. At this point, it should be mentioned that for the end user of the system, each service constitutes the set of content with specific attributed, in regard to the topic that each of these services is meant to support. However, the content, the information and the functionality that each user can access constitute only the final point of the informational system. Thus, there is a whole life cycle that need to be followed so as a service will be operated effectively. This life circle is the following:

- Definition of the objectives and the theme of each service.

- Definition of the content types for each of the services.

- Definition of the functionalities for each of the services.

- Definition of the roles and the access rights that each roles involves for each of the provided services and functionalities.

- Definition of the available working groups in the scope of each service.

\subsection{Services}

After defining the framework of the informational system, the step that followed was to actually define and describe the services and functionality that the system would offer, as these were extracted from the employees and citizens needs. From the interviews and 
questionnaires, the following categories of services were extracted as critical for the success of the Unified Informational System:

- Personalised Access Services. The Personalised Access Services concern the basic operations needed for the personal support of all the users registered to the system. This service includes the register and login procedure as well as the personal details service. The user has to be registered to be able to use the advanced functionality of the system. In addition, whenever the user wants to access the system he/she has to login. This procedure is repeated whenever the user wants to make a critical change to the system.

- Back-Office Services. The Back-Office Services are related with the administration of basic entities of the system. In particular, these services concern the operations related with the creation and administration of the basic structure of the system and the initialisation of the supported services. Almost every informational system has one or more administrators who are responsible for the good functioning of the procedures of the system and for the integrity of the content.

- Content Management Services. The Content Management Services concern, mainly, the actions that can be applied on each of the instances of the content items. The content management services contain the services provided to the users that can help me interact with information objects. This can be papers (text), multimedia, code, etc.

- Internal Organisational Services. The Internal Organisational Services simulate the processes of a real organisation and contribute to the effective structuring and monitoring of all information necessary for the effective, secure and smooth operation of the party. This simulation helps the user to realise the hierarchy of the organisation, its role and the responsibilities of each employee during a procedure.

- Information and Participation Services. The Information and Participation Services provide to the users of the system the ability to get information on various topics that are related with the party of PASOK, as well as to express their opinions and ideas on certain issues. The information services usually rely on papers from speeches, newspaper, media and the internet. The members of such a service do not have to search the web for material concerning a variety of issues, but they collect all the desired information from this service. In addition, the users can add their own information and comment or discuss them for the promotion of democracy through the system.

- Communication and Collaboration Services. The Communication and Collaboration Services define a set of interrelated functionalities that can be independent from the texture of an application or functionality. They are exploited for the support of the communication and collaboration of the system's user both in synchronous and asynchronous way.

\section{Technical specifications}

The next step was to investigate a technological approach and finally a technological solution. This step includes the definition of the technical requirements, the criteria for 
the selection of the technical solutions as well as the technology monitoring. Furthermore, this step includes the definition of a unified technological solution and the design of the system architecture.

\subsection{Requirements, criteria and technology monitoring}

At this point, it was important to define the specifications of the proposed system, as these were extracted from the questionnaires, the interviews and the discussions conducted, as well as from the design of the system. These basic requirements that define the specifications of the proposed technological solution could be summarised as follows:

- A Unified Technological Platform for the development and disposal of new services and integration/adaptation of the existent application to the new unified framework.

- Modelling and semantic connection of the imported information.

- Easy collection and modelling of external resources.

- Prompt and cost-effective creation of new services and electronic version of the modelled information with an effective and automatic way and with the adaptation of a unified branding.

- The Unified Technological Platform should be based on open standards and open source software tools. Open Source Software constitutes an indication of an open platform, transparent to its processes and functionality, permits an easier and quicker parameterisation and adaptation to the needs that need to be met.

- Access to all services through different devices, based on the web (web-enabled services/applications): Desktop Web Browser, Mobile Phone, Palmtop.

- Ability for information provision in multiple different forms: HTML, XHTML, CHTML (imode), SMS, VRML, VoiceML, Word, Excel, PDF, Image.

- Access through information pull and push (e.g., electronic magazine, SMS, etc.).

- Unified User Interface for all the services and applications.

- Single sign-on for access to all of the provided services with a unified rights management, powerful safety mechanisms and unification of internet and intranet applications. This involves unified users' management, user modelling and integration of all information resources for the users in a unified database system.

- Personalised access according to user's access rules and rights. There is an automatic or selected adjustment of the User Interface and services in the users' profiles.

As it has become clear, e-PASOK is characterised from different components, which results in a wide range of available technologies that can be exploited for the development and support of the desired functionality. As it can be extracted from the above, in general terms, the informational system will be composed of a basic platform, on the top of which the following services will be integrated:

- A Content Management System (CMS)

- A Citizen Relationship Management (CRM) and Collaboration system with the citizens/members and cooperators of PASOK. 
Therefore, for each of the above components, a technology monitoring has been realised, so as to obtain a concrete view on the available technologies and tools, which in turn will facilitate the decision of the technologies, the standards and tools that will be adopted for the optimum development of the proposed system.

\subsection{Proposed solution and system architecture}

This section is engaged with the description of the main characteristics of the technological framework as well as the system architecture that the system will be based on (Figure 4). The proposed framework is selected to be consistent with the development requirements and the need to avoid highly cost demanding implementation and support of the provided services. The basis of the framework will be constituted by open standards, APIs, tools and development environments that are based on Java (J2SE) and J2EE technology.

Figure 4 System architecture (see online version for colours)

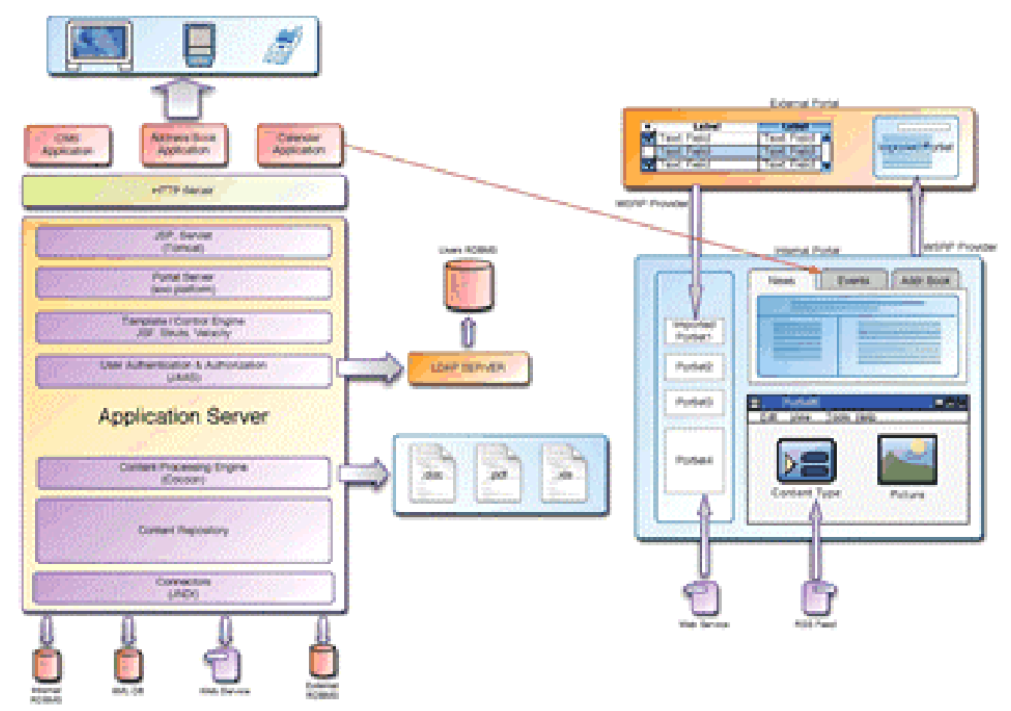

As far as it concerns the software component that will be adopted for the structure of the Main Technological Platform, the need for open source solutions in combination with the above-mentioned proposal of standards leads to the implementation of the technological platform with the following components:

- LINUX Operating System for the installation of all technologies and applications.

- SUN's J2SE SDK for Java development.

- JBOSS Application Server, as the certified implementation platform of J2EE standard (web container, EJB Container, JMS implementation, JNDI services, JAAS implementation, etc.). 
- Portal Server ExoPlatform as the implementation platform of the Portal Services and Portlets Container. The system will offer the support of single sign on operation and presentation/access to the provided applications, through a unified User Interface that will support personalisation of the users' needs.

- $\quad$ Postgres Database Server for supporting back-end storage services.

- OpenLDAP Server for the development of Directory Services. Both the modelling and storage of the overall information for the users will be performed in the database server. A small part of this information will be replicated to the OpenLDAP Server. This part will constitute the information required for user authentication as well as for user access to the provided services.

Regarding the CMS application, the basic software component of CMS will be adopted, that is a Content Repository application. The content will be stored in a relational database, while the administration will be performed by this application as well. In addition, the content presentation will be performed through a templating engine. The content will be retrieved from the storage and will be presented through the portal environment. The content publication will be performed through XML standards and XSLT converters. The same storing application will also manage the life cycle of the content, implementing the workflows that are linked to the content. Furthermore, applicable methods and procedures will be developed, which, supported by the CMS application, will allow the systematic production, collection, management and publication of content.

The main concept behind the proposed solution is the creation and collection of content in a way that it can be reused in multiple versions. The content management will be performed with a unified and central way in a content repository, where the different versions will be updated. Thus, economy in the production and maintenance of the content in it various stages of its life cycle is achieved. Finally, the structuring and standardisation of the content repository results in added value as it allows the automatisation of content provision.

The content representation application is proposed to be implemented using the Apache Struts library and the Java Server Faces standard. This technology allows the development of a complete thin client web-based application. These technologies allow the operability of the application to environments like i-mode mobile phone or WAP.

\section{Conclusions}

The main objective of this paper was to propose a methodology for designing an informational system for political parties to provide e-government and e-democracy services. Furthermore, this paper presented all the steps followed for the design of e-PASOK, which is a Unified Framework for the Collection, Management and Disposition of Content for the party of PASOK. The design of this system is based on the needs of the users and the advanced tools and solutions that technology can offer. 


\section{Future work}

Our next step is the systematic implementation of e-PASOK. The implementation plan is based on three main phases:

- modular analysis and implementation of the system services

- $\quad$ step-by-step delivery of the services and internal evaluation

- final services delivery and users' training on the usage of the system.

Based on the above, PASOK, prioritising the need for the above-mentioned services, plans future projects that involve, among other things:

- the incubation of a more sophisticated open-ended dialogue, for the year to come, through the introduction of thematical - 'professional' facilitation, encouraging the formation of citizens' networks and use of slashdot like tools

- the use of e-voting techniques for the election of candidates, representing PASOK, during next years' Municipal Elections

- a nationwide training programme for political activists on internet's basics

- the development of an internet-based information system, providing access to online information services, CRM and e-consultation services.

\section{Acknowledgements}

We thank all of PASOK's employees for their valuable help and contribution they provided.

\section{References}

Benjamin, R. and Levinson, E. (1993) 'A framework for managing IT-enables change', Sloan Management Review, Vol. 34, No. 4, pp.23-33.

Garcia-Arribas, G. and Lopez-Crespo, F. (2003) 'Landscape of e-Government at the dawn of the 21st century', Upgrade-The European Journal for the Informatics Professional, Vol. IV, No. 2, April, pp.2-6.

Kubicek, H., Westholm, H. and Winkler, R. (2003) 'Prisma Strategic Guideline 9- eDemocracy', Information Societies Technologies (IST) Programme, http://www.prisma-eu.net/deliverables/ SG9DEMOCRACY.pdf

Layne, K. and Lee, J. (2001) 'Developing fully functional e-government: a four stage model', Government Information Quarterly, Vol. 18, pp.122-36.

Liikanen, E. (2003) 'E-Government and the European Union', Upgrade-The European Journal for the Informatics Professional, Vol. IV, No. 2, 7-10.

Musgrave, S. (2004) 'The community portal challenge - Is there a technology barrier for local authorities?', Telematics and Informatics, Vol. 21, No. 3, pp.261-272.

Pizarro, N. and De La Torre, P. (2003) 'e-Government and the network society', Upgrade - The European Journal for the Informatics Professional, Vol. IV, No. 2, April, 14-20.

Poullet, Y. (1998) 'Freedom and information highways or how to ensure electronic democracy', Telematics and Informatics, Vol. 15, No. 3, pp.163-180. 